\title{
A REMARK ON FINITE GROUPS ${ }^{1}$
}

I. N. HERSTEIN

In this paper we prove a theorem about finite groups which, although it is natural and its proof rather simple, seems to be new and nonexistent in the lierature.

The object of this note is to prove the following

Theorem. Let $G$ be a finite group, $A$ an Abelian subgroup of $G$. If $A$ is a maximal subgroup of $G$ then $G$ is solvable.

If $G$ is a finite group and $H$ is a subgroup of $G$ then we say that $H$ is a Frobenius subgroup of $G$ if $x H x^{-1} \cap H \neq(1)$ if and only if $x \in H$.

Concerning such Frobenius subgroups there is a celebrated theorem due to Frobenius [1] which asserts: let $T=\left\{a \in G \mid a \oplus x H x^{-1}\right.$ all $x \in G$ or $a=1\}$; then $T$ is a normal subgroup of $G$. We call it the complementary subgroup of $H$.

Suppose now that a finite group $G$ has an automorphism $\phi$ leaving only 1 fixed. Thus the elements $x^{-1} \phi(x)$ are all distinct as $x$ ranges over $G$, so they must fill out all of $G$. Let $p$ be a prime which divides the order of $G$ and let $S_{p}$ be a $p$-Sylow subgroup of $G$. Then $\phi\left(S_{p}\right)$ is also a $p$-Sylow subgroup of $G$ and so $\phi\left(S_{p}\right)=y S_{p} y^{-1}$ for some $y \in G$. Pick $x \in G$ so that $y^{-1}=x^{-1} \phi(x)$. Then as can immediately be verified, $\phi\left(x S_{p} x^{-1}\right)=x S_{p} x^{-1}$. That is, for each prime $p$ there is a $p$-Sylow subgroup $S_{p}$, say, of $G$ left set-wise invariant by $\phi$. We claim that $S_{p}$ is unique in this regard. For if $N\left(S_{p}\right)=\left\{x \in G \mid x S_{p} x^{-1}=S_{p}\right\}$ then it is clear that $\phi\left(N\left(S_{p}\right)\right)=N\left(S_{p}\right)$. Since $N\left(S_{p}\right)$ is invariant under $\phi, \phi$ induces an automorphism on $N\left(S_{p}\right)$ leaving only 1 fixed, whence every element in $N\left(S_{p}\right)$ can be written in the form $n^{-1} \phi(n)$ with $n \in N\left(S_{p}\right)$. Thus if $a^{-1} \phi(a) \in N\left(S_{p}\right)$, then $a^{-1} \phi(a)=n^{-1} \phi(n)$ for some $n \in N$ and so $a=n$ from which, of course, we have that $a \in N$. Now suppose $\phi\left(S_{p}\right)=S_{p}$ and $\phi\left(S_{p}^{\prime}\right)=S_{p}^{\prime}$ for some other $p$-Sylow subgroup. Since $S_{p}^{\prime}=a S_{p} a^{-1}$ for some $a \in G, a S_{p} a^{-1}=S_{p}^{\prime}=\phi\left(S_{p}^{\prime}\right)=\phi\left(a S_{p} a^{-1}\right)$ $=\phi(a) S_{p} \phi(a)^{-1}$, leading to $a^{-1} \phi(a) \in N\left(S_{p}\right)$, and so $a \in N\left(S_{p}\right)$ and finally to $S_{p}^{\prime}=a S_{p} a^{-1}=S_{p}$. Thus $S_{p}$ is indeed unique with respect to being left invariant by $\phi$.

If $\psi$ is an automorphism of $G$ which commutes with $\phi$ then since $\phi\left(S_{p}\right)=S_{p}, \phi \psi\left(S_{p}\right)=\psi \phi\left(S_{p}\right)=\psi\left(S_{p}\right)$, and so, since $\psi\left(S_{p}\right)$ is a $p$-Sylow subgroup left fixed by $\phi, \psi\left(S_{p}\right)=S_{p}$. Thus if $\mathfrak{A}$ is an Abelian group of

Received by the editors October 18, 1957.

1 The research reported in this paper was done at the 1957 Summer Mathematical Conference at Bowdoin College, under contract to the Electronics Research Directorate, Air Force Cambridge Research Center, Air Research and Development Command, Contract Number AF19-604-2226. 
automorphisms of $G$ where every automorphism in $\mathfrak{A}$ acts on $G$ in a manner leaving only 1 fixed then there is a unique $p$-Sylow subgroup, for each prime $p$, of $G$ left invariant by all the elements of $\mathfrak{A}$.

We summarize all this into the

Lemma. Let $\mathfrak{A}$ be an Abelian group of automorphisms acting on the finite group $G$ so that for every $\phi \in \mathfrak{U}$ whenever $\phi(x)=x$ then $x=1$. Then for every prime $p$ dividing the order of $G$ there is a unique $p$-Sylow subgroup of $G$ which is taken into itself by all the elements of $\mathfrak{A}$.

We are now ready to proceed with the proof of the theorem.

Proof of the Theorem. We proceed by induction over the order of $G$. If $N(A)=\left\{x \in G \mid x A x^{-1} \subset A\right\}$ is different from $A$, since it is a subgroup of $G$ and contains $A$, by the maximality of $A$ we would be lead to $N(A)=G$. But then $A$ is a normal subgroup of $G$. Since $A$ is maximal in $G, G / A$ has no nontrivial subgroups so must be of prime order, hence cyclic and so solvable. Since $A$ is Abelian and thus solvable and since $G / A$ is solvable this all results in the solvability of $G$.

If, on the other hand, $N(A)=A$ consider $W=x A x^{-1} \cap A$ for $x \notin A$. If $W \neq(1)$ let $w \neq 1 \in W$. Consider $Z(w)=\{x \in G \mid x w=w x\} . Z(w)$ is a subgroup of $G$ and since $A$ is Abelian and $w \in A$, it contains $A$; since $w \in x A x^{-1}, Z(w)$ also contains $x A x^{-1}$ which is different from $A$ since $x \notin A=N(A)$. Thus $Z(w)=G$, and so $w$ is in the center of $G$. The subgroup (w) generated by $w$ is normal in $G$ and is contained in $A$. By our induction, then, $G /(w)$ is solvable and since $(w)$ also is, it follows that $G$ is solvable.

We are left with only one possibility to consider, namely the situation where $N(A)=A$ and where $x A x^{-1} \cap A=(1)$ if $x \in A$. Thus $A$ is a Frobenius subgroup of $G$. Let $T$ be the complementary subgroup of $A$ in $G$. Since $T$ is normal in $G$ for all $a \in A, a T a^{-1}=T$. Thus each $a \in A$ induces an automorphism $\tau_{a}$ on $T$. We claim that for $a \neq 1$, $\tau_{a}(t)=t$ for $t \in T$ forces $t=1$. For if $\tau_{a}(t)=t$ then $a t a^{-1}=t$ and so $t^{-1} a t=a \in A \cap t^{-1} A t=(1)$ if $t \in A$. Thus $t=1$ follows. Thus by our lemma there is a $p$-Sylow subgroup $S_{p}$ of $T$ left fixed by every $\tau_{a}$ for $a \in A$. That is, $a S_{p} a^{-1}=S_{p}$ for all $a \in A$. Hence $N\left(S_{p}\right) \supset A$, and it contains $S_{p} \nsubseteq A$. So $N\left(S_{p}\right)=G$ whence $S_{p}$ is normal in $G$. We claim $S_{p}=T$. For, since $S_{p}$ is normal in $G, A S_{p}$ is a subgroup of $G$ and contains $A$ properly. So $A S_{p}=G$. Thus if $t \in T, t=a s$ with $a \in A$, $s \in S_{p} \subset T$. But then $a=t s^{-1} \in T \cap A=(1)$ from which we have that $t=s$ and so $T=S_{p}$. Since $G / T$ is isomorphic to $A$ which is solvable (Abelian) and $T=S_{p}$ is nilpotent, $G$ must be solvable.

The following corollary is an extension of an unpublished result of 
Kaplansky and the present author.

Corollary to the Theorem. Let $G=A B A$ be a finite group where $A$ is an Abelian subgroup, $B$ a subgroup of prime order. Then $G$ is solvable.

Proof. Suppose $A$ is not a maximal subgroup of $G$. Then it is contained in a subgroup $C \neq A, C \neq A$. Thus there is an $x=a_{1} b^{k} a_{2}$, with $b^{k} \neq 1$, in $C$ where $a_{1}, a_{2} \in A$ and where $b$ generates $B$. Since $C \supset A$ we get that $b^{k} \in C$, and so $b \in C$ since $b$ is of prime order. Since $C \supset A$ and $C \supset B, C \supset A B A=G$, a contradiction. Consequently $A$ is a maximal subgroup of $G$ and the result is immediate from our theorem.

\section{BiBLIOGRAPHY}

1. A. Speiser, Theorie der Gruppen von Endlicher Ordering, 2d ed., Springer, 1927. Cornell University 